\title{
Optimal Topology for an Intra-Vehicle Antenna Implemented on Dashboard Foams
}

\author{
Freek Boeykens*, Quinten Doolaege, Frederick Declercq and Hendrik Rogier \\ Ghent University, Department of Information Technology (INTEC) \\ Ghent, Belgium \\ Email: freek.boeykens@intec.ugent.be
}

\begin{abstract}
This paper presents several antenna topologies for intra-vehicle communication in the unlicensed $2.45 \mathrm{GHz}$ ISM band $(2.4 \mathrm{GHz}-2.485 \mathrm{GHz})$. The antennas are implemented using flexible foam materials compatible with the production process of dashboards, as dashboards offer a large platform for easy and unobtrusive integration. In order to obtain the optimal intravehicle antenna, the different topologies are compared in terms of antenna gain, efficiency, AR bandwidth and AR robustness against small fabrication errors.
\end{abstract}

\section{INTRODUCTION}

The rising interest in wireless intra-vehicle communication leads to the concept of the Cable Free Car. Within this concept it becomes possible to connect certain wireless devices such as PDAs and GSMs to a central infotainment console of the car. These connections use protocols such as Bluetooth, ZigBee or Wifi, which operate in the license-free $2.45 \mathrm{GHz}$ ISM band $(2.4 \mathrm{GHz}-2.485 \mathrm{GHz})$. An unobtrusively and invisibly integrated antenna completely covering this ISM band is thus required and past research taught us that this antenna is optimally positioned in the dashboard with the main lobe of the radiation pattern towards the center of the car [1]-[3].

The optimal antenna for this application must satisfy three main requirements:

1) easy integration in the dashboard;

2) shock and stress resistance;

3) quasi-circular polarisation.

The first requirement leads to the use of patch antennas. These low-profile planar antennas are light in weight, compact and easy to fabricate in mass production. Also flexible materials have to be used, since extra effort would be needed for incorporating a rigid antenna in the flexible dashboard.

According to the second requirement, the use of vias should be avoided because these connections are too fragile for this environment. Therefore, an aperture-coupled feed structure is proposed. Such a structure has no physical connection between the feed line and the patch, making the antenna shock and stress resistant. It also allows to use different materials as antenna and feed substrate, minimising back radiation [4]. Moreover, an active circuit can easily be integrated onto the feed substrate.

In order to satisfy the last requirement, different antenna topologies exist in literature. In this paper, several topologies are investigated and compared in terms of antenna gain,
TABLE I

SUBSTRATES FOR ALL ANTENNAS.

\begin{tabular}{|c||c|c|c|}
\hline Type & Description & $\epsilon_{r}$ & $\tan \delta$ \\
\hline \hline Feed substrate & $\begin{array}{c}\text { Two-layer complex aromatic } \\
\text { + aliphatic polyurethane }\end{array}$ & 2.53 & 0.065 \\
\hline Antenna substrate & $\begin{array}{c}\text { Aromatic polyurethane } \\
\text { with blown air bubbles }\end{array}$ & 1.45 & 0.03 \\
\hline
\end{tabular}

TABLE II

DESIGN PARAMETERS FOR TOPOLOGY A AND ITS MODIFICATIONS.

\begin{tabular}{|c||c|c|c|}
\hline Parameter & A $[\mathrm{mm}]$ & A. $1[\mathrm{~mm}]$ & A.2 $[\mathrm{mm}]$ \\
\hline \hline Patch $(\mathrm{L} \times \mathrm{W})$ & $44.5 \times 41.9$ & $46.4 \times 43.7$ & $41 \times 41$ \\
\hline Slot $(\mathrm{L} \times \mathrm{W})$ & $18 \times 2$ & $14.2 \times 1.4$ & $17.2 \times 1.5$ \\
\hline Stub & 8.27 & 8.57 & 10 \\
\hline Feed & 2.8 & 2.8 & 2.8 \\
\hline Dogbone (l) & - & 5 & - \\
\hline Slit (h) & - & - & 6.25 \\
\hline Slit (v) & - & - & 8.25 \\
\hline
\end{tabular}

efficiency and axial ratio (AR) bandwidth. Also the ARrobustness is evaluated when small fabrication errors are introduced.

Section II describes the used materials and the different antenna topologies. Section III presents the simulation results. The conclusions are drawn in Section IV.

\section{TOPOLOGIES}

All antenna topologies in this Section use the same antenna and feed substrates. These flexible substrates were provided by Recticel $^{\circledR}$ and are typically used in the production process for dashboards. A description of the materials and their characteristics is shown in Table I.

The rest of this Section discusses the tested topologies. These topologies were simulated with the 2.5D full-wave field simulator ADS Momentum ${ }^{\circledR}$ and optimised by imposing the following design criteria for the reflection coefficient $\left(S_{11}\right)$ and the AR in the $2.45 \mathrm{GHz}$ ISM band:

$$
\begin{aligned}
\left|S_{11}\right| & <-10 \mathrm{~dB} \\
\mathrm{AR} & <3 \mathrm{~dB} .
\end{aligned}
$$

As a result of our design procedure, all topologies satisfy (1) in the entire ISM band. The AR will be discussed in Section III. 


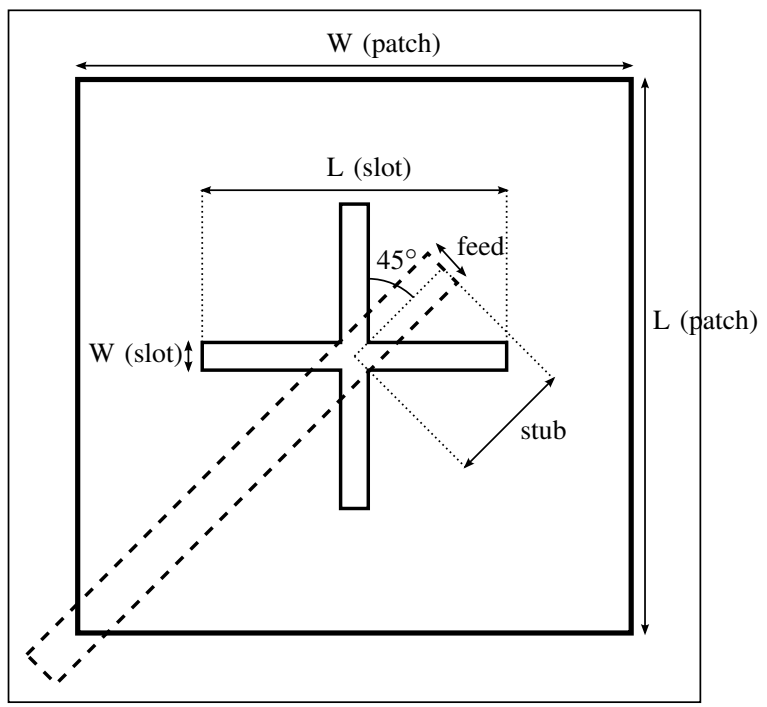

(a)

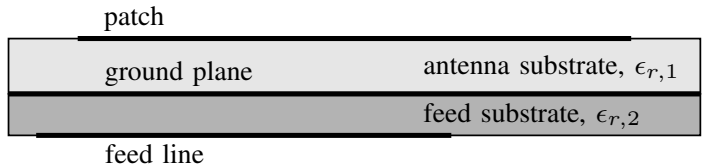

(b)

Fig. 1. Topology A: top view (a) and side view (b).

\section{A. Crossed slot antenna with single feed line}

The first antenna topology, depicted in Fig. 1, consists of a nearly-square patch, a crossed slot and a feed line placed along the diagonal of the cross. Quasi-circular polarisation is obtained by excitation of two orthogonal modes in the crossed slot [5], [6]. The design parameters are shown in Table II.

Two modifications to this topology were also simulated (their design parameters can be found in Table II as well):

1) Dogbones: Here, the aperture is extended with socalled 'dogbones', making the crossed slots $\mathrm{H}$-shaped. These dogbones increase the coupling between the feed line and the patch due to a more uniform equivalent magnetic field distribution on the slot [7]. This modification is shown in Fig. 2(a).

2) Patch with slits: A second modification introduces slits in the patch in order to obtain a reduced patch size and a broader AR bandwidth [8]. This topology is depicted in Fig. 2(b).

\section{B. Crossed slot antenna with single series feed}

This antenna also couples the signal through a crossed slot, but unlike previous topologies, a series feed line is placed behind the aperture. Between each arm of the aperture is a quarter-wavelength section of the feed line positioned in order to obtain circular polarisation. This has the effect of hybrid feeding techniques and thus widens the AR bandwidth [9]. The topology is shown in Fig. 3 and the design parameters are presented in Table III.

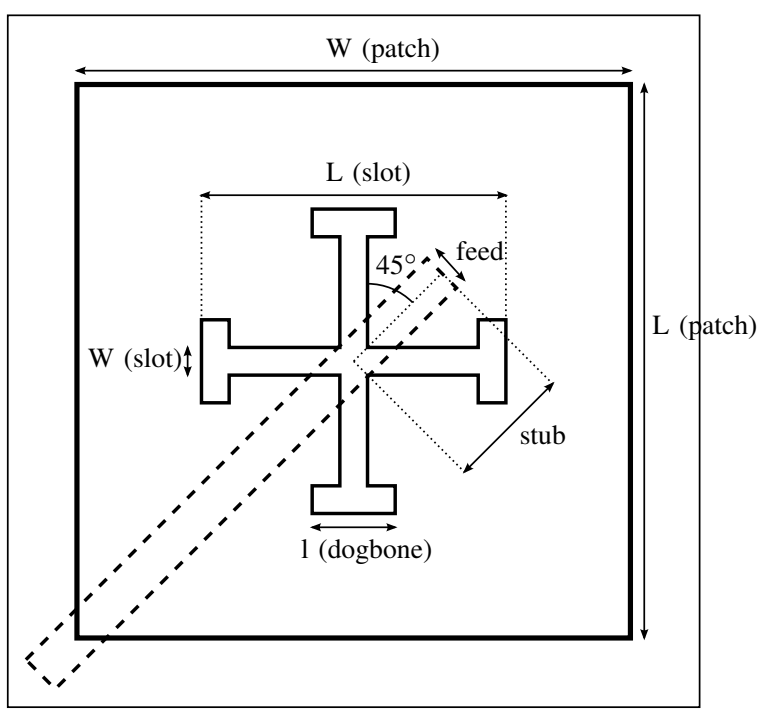

(a)

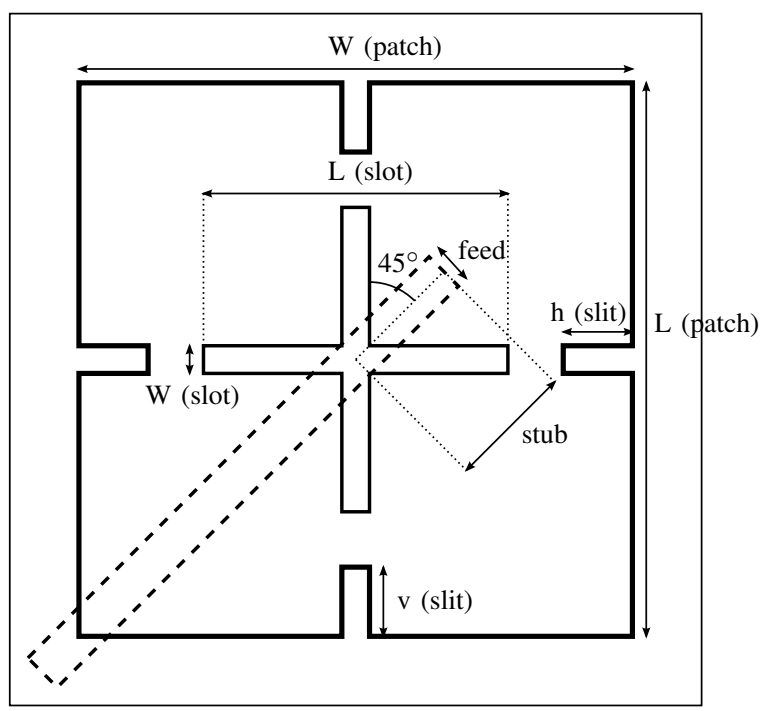

(b)

Fig. 2. Modifications to topology A (top view): dogbones (a) and patch with slits (b).

TABLE III

DESIGN PARAMETERS FOR TOPOLOGY B

\begin{tabular}{|c||c|}
\hline Parameter & Value $[\mathrm{mm}]$ \\
\hline \hline Patch $(\mathrm{L} \times \mathrm{W})$ & $40.5 \times 40$ \\
\hline Slot $(\mathrm{L} \times \mathrm{W})$ & $26 \times 1$ \\
\hline Stub & 12.5 \\
\hline Feed & 2.8 \\
\hline$\lambda / 4$ & 18.2 \\
\hline
\end{tabular}

\section{Quadrature hybrid coupler}

The last topology that is tested uses a quadrature hybrid coupler for circular polarisation. If one port of this 4-port structure is terminated by its characteristic impedance, then the coupler acts as a power splitter with two output signals equal in amplitude and with $90^{\circ}$ phase difference. Now two 


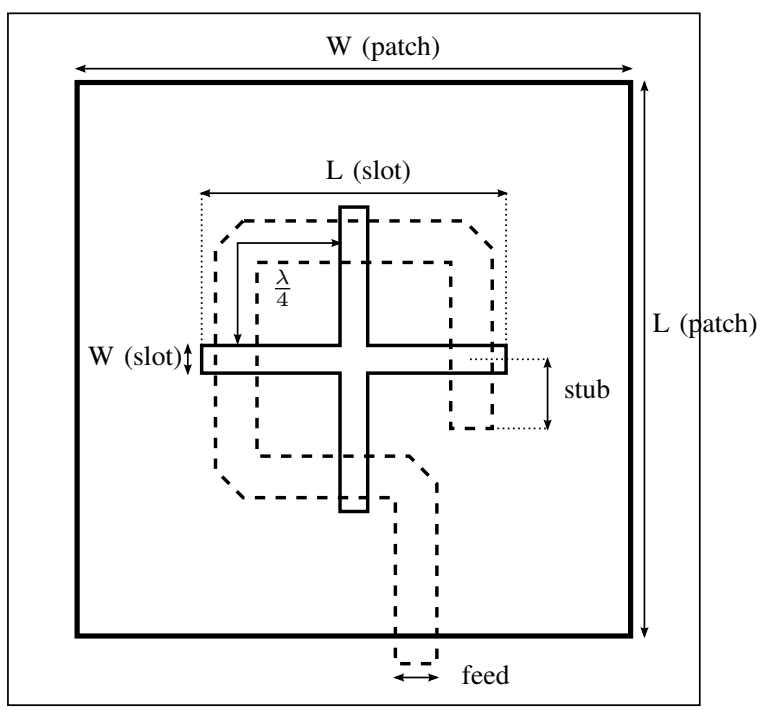

Fig. 3. Topology B: top view.

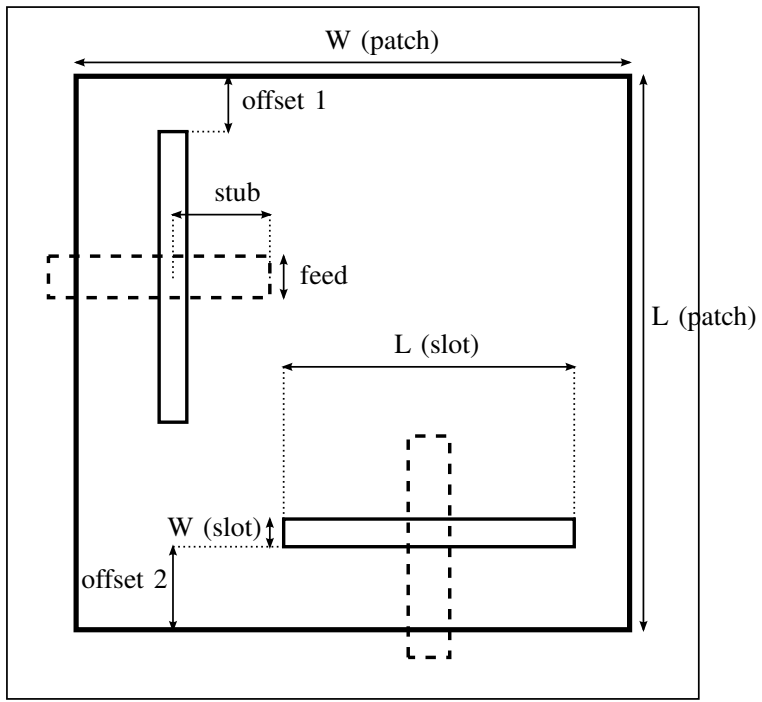

Fig. 4. Topology C: top view (hybrid coupler not shown).

separate rectangular apertures couple the power on the two feed lines into the patch.

The use of a hybrid coupler makes the design more complex, but ensures circular polarisation in the complete ISM band [10]. Fig. 4 depicts this topology and Table IV shows the design parameters.

\section{Simulation RESUlts}

\section{A. Antenna gain and efficiency}

Table $\mathrm{V}$ shows the simulated antenna gain and efficiency in broadside direction at $2.45 \mathrm{GHz}$. From this point of view, topology A would be the best fit, as it has the highest gain and efficiency. The lowest gain and efficiency can be found for topology C.
TABLE IV

DESIGN PARAMETERS FOR TOPOLOGY C.

\begin{tabular}{|c||c|}
\hline Parameter & Value $[\mathrm{mm}]$ \\
\hline \hline Patch $(\mathrm{L} \times \mathrm{W})$ & $46.3 \times 46.3$ \\
\hline Slot $(\mathrm{L} \times \mathrm{W})$ & $20 \times 3$ \\
\hline Stub & 10.1 \\
\hline Feed & 2.8 \\
\hline Offset 1 & 4.85 \\
\hline Offset 2 & 5.35 \\
\hline
\end{tabular}

TABLE V

Simulated GAin AND EFFICIENCY OF ALL TOPOLOGIES AT $2.45 \mathrm{GHZ}$.

\begin{tabular}{|c||c|c|}
\hline Topology & Gain [dBi] & efficiency [\%] \\
\hline \hline A & 4.018 & 41.3 \\
\hline A with dogbones & 4.005 & 38.7 \\
\hline A with slits & 3.376 & 35.3 \\
\hline B & 3.153 & 39.6 \\
\hline C & 2.923 & 29.7 \\
\hline
\end{tabular}

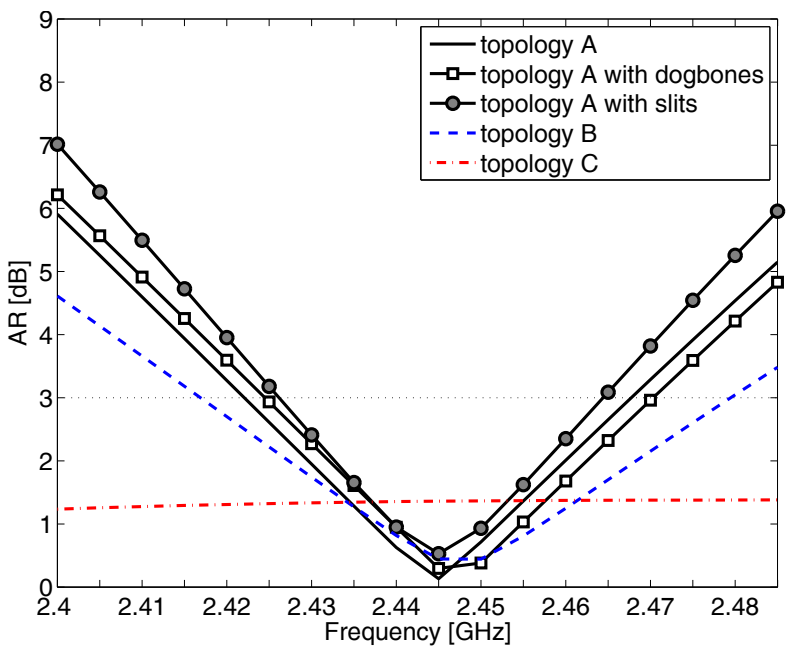

Fig. 5. Simulated AR in the $2.45 \mathrm{GHz}$ ISM band for all topologies.

\section{B. AR performance}

Another important criterion for the intra-vehicle antenna is quasi-circular polarisation in the ISM band. As a rule of thumb, circular polarisation is assumed for an AR lower than $3 \mathrm{~dB}$.

Fig. 5 demonstrates the AR in the $2.45 \mathrm{GHz}$ ISM band for all topologies. One can see that the curves for topology A and topology A with dogbones are almost the same. Introducing slits in the patch leads to a worse AR bandwidth and the highest values at the ISM band edges. Topology B has a broader bandwidth and lower values at the boundaries of the ISM band and for topology $\mathrm{C}$, there is circular polarisation in the entire ISM band.

A sensitivity analysis is also performed by introducing small fabrication errors on the designs and evaluating the $A R$ in the ISM band. Only the errors on the apertures are shown, since they affect the AR performance the most. The introduced 
errors can be divided into two parts:

1) Alignment errors: Alignment errors can be simulated by moving the slots in all directions. As can be seen on Fig. 6(a), the $3 \mathrm{~dB}$ AR bandwidth remains almost constant for all topologies when the slots are shifted by $1 \mathrm{~mm}$ to right. For topology A and its modifications, moving the slots only provokes a shift of the curves. The AR curve of topology B has moved upwards, but the values at the edges of the ISM band are more or less the same as without alignment errors. Topology $\mathrm{C}$ is still circularly polarised in the entire ISM band.

Similar results are obtained when the slots are moved in the other directions. All topologies also still satisfy (1) after introduction of aligment errors.

2) Slot dimension errors: The slot dimension errors are simulated by enlarging and widening the apertures. The largest effect can be observed for an asymmetrical enlargement of the slots, as shown in Fig. 6(b), which depicts the AR of all topologies after an enlargement of the vertical apertures by $1 \mathrm{~mm}$. Unlike alignment errors, the slot dimension errors introduce a decrease in AR bandwidth (except for topology C, which remains circularly polarised in the entire ISM band). Also, the return loss of topology B only satisfies (1) for frequencies between $2.46 \mathrm{GHz}$ and $2.485 \mathrm{GHz}$.

When the vertical apertures enlarge even more, the AR curves of topologies A and B stay above $3 \mathrm{~dB}$ and hence there is no frequency in the $2.45 \mathrm{GHz}$ ISM band anymore for which these topologies are circularly polarised.

The results for other slot dimension errors have less influence on the AR curves, so we can conclude that asymmetrical slot dimensions lead to the worst AR performances.

\section{CONCLUSION}

In the search for an optimal intra-vehicle antenna, several topologies were investigated and compared. Topology $\mathrm{C}$ is definitely the most robust of all topologies against fabrication errors. However, circular polarisation is not necessary for intravehicle communication, since the purpose here is to minimise the polarisation mismatch between transmitter and receiver. Therefore it is recommended to use a simpler design with a higher gain and efficiency and hence we suggest topology A as the optimal intra-vehicle antenna.

\section{ACKNOWLEDGMENT}

The authors would like to thank Recticel ${ }^{\circledR}$ for the provision of the dashboard materials.

The work of F. Boeykens was supported by a doctoral grant from the Agency for Innovation by Science and Technology in Flanders (IWT).

\section{REFERENCES}

[1] H.-M. Tsai, C. Saraydar, T. Talty, M. Ames, A. Macdonald, and O.K. Tonguz, ZigBee-based Intra-car Wireless Sensor Network, IEEE Int Conf on Comm, pp. 3965-3971, 2007.

[2] R. de Francisco, L. Huang, G. Dolmans, H. de Groot, Coexistence of ZigBee wireless sensor networks and Bluetooth inside a vehicle, IEEE Int Symp on Personal, Indoor and Mobile Radio Comm, pp. 2700-2704, 2009.

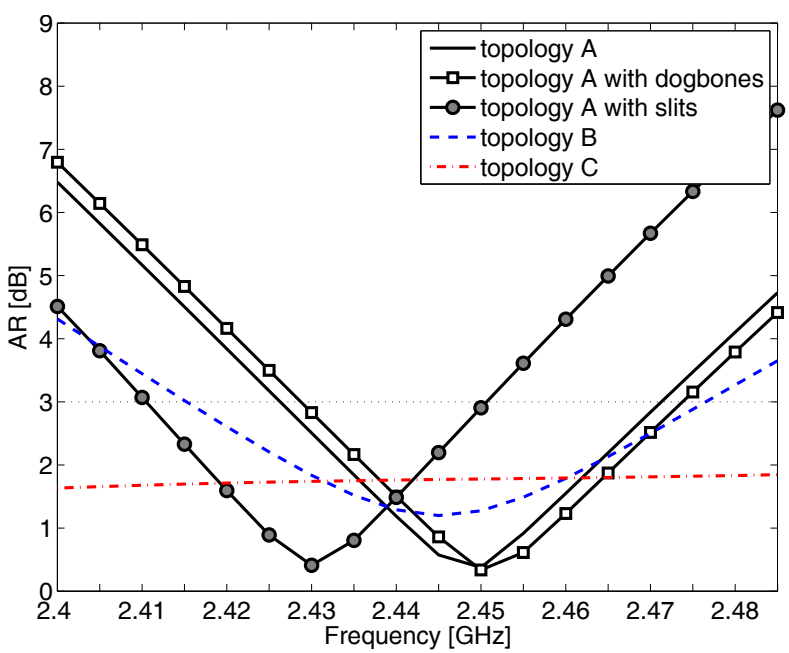

(a)

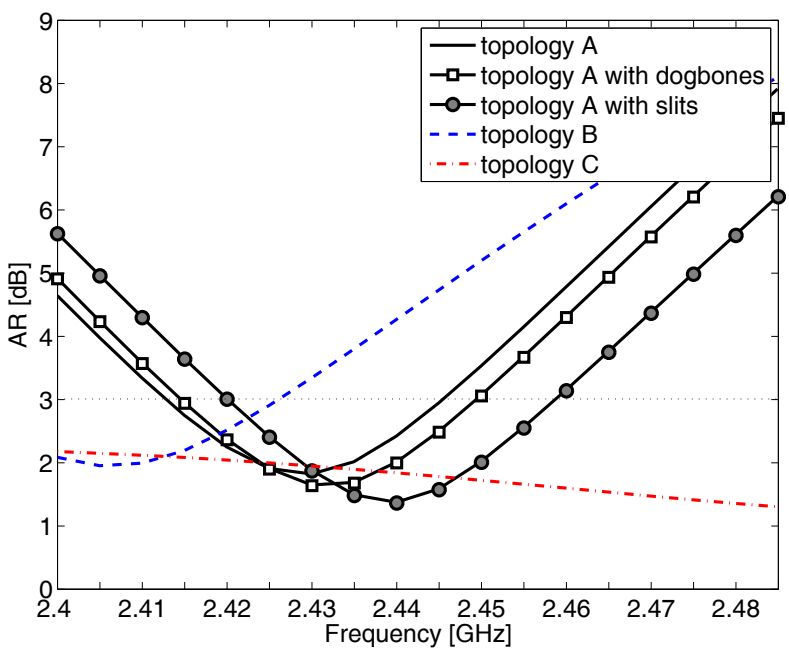

(b)

Fig. 6. AR performance of all topologies in the $2.45 \mathrm{GHz}$ ISM band: shift of the slot to the right by $1 \mathrm{~mm}$ (a) and enlargement of the vertical slot with $1 \mathrm{~mm}$ (b).

[3] F. Boeykens, Q. Doolaege, F. Declercq, H. Rogier, A planar aperturecoupled patch antenna implemented on dashboard foams for intravehicle communication, accepted for publication in Microwave and Optical Technology Letters.

[4] D.M. Pozar, Microstrip antenna aperture-coupled to a microstrip line, Electronics Letters, vol. 21, no. 2, pp. 49-50, 1985.

[5] T. Vlasits, E. Korolkiewicz, A. Sambell, B. Robinson, Performance of a cross-aperture coupled single feed circularly polarised patch antenna, Electronics Letters, vol. 32, no. 7, pp. 612-613, 1997.

[6] B. Al-Jibouri, H. Evans, E. Korolkiewicz, E.G. Lim, A. Sambell, T.Viasits, Cavity model of circularly polarised cross-aperture-coupled microstrip antenna, IEEE Proc-Microwave Antennas Propag, vol. 148, no. 3, pp. 147-152, 2001.

[7] D. Pozar, S. Targonski, Improved coupling for aperture coupled microstrip antennas, Electronic Letters, vol. 27, no. 13, pp. 1129-1131, 1991.

[8] K.L. Wong, J.-Y. Wu, Single-feed small circularly polarised square microstrip antenna, Electronics Letters, vol. 33, no. 22, pp. 1833-1834, 1997

[9] H. Kim, B.M. Lee, Y.J. Yoon, A single-feeding circularly polarised microstrip antenna with the effect of hybrid feeding, IEEE Ant. and Wireless Propag. Letters, vol. 2, pp. 74-77, 2003.

[10] D. Pozar, Microwave Engineering, Addison Wesley, 1990. 